\title{
SOME RELATIONS ASSOCIATED WITH AN EXTENSION OF KOSHLIAKOV'S FORMULA
}

\section{KUSUM SONI}

It is known that the following three relations are equivalent:

(1) Functional equation for $\zeta^{2}(s)$

$$
\pi^{-s}\left[\Gamma\left(\frac{s}{2}\right)\right]^{2} \zeta^{2}(s)=\pi^{s-1}\left[\Gamma\left(\frac{1-s}{2}\right)\right]^{2}[\zeta(1-s)]^{2}
$$

(2) Koshliakov's formula

$$
\begin{aligned}
\gamma-\log \left(\frac{4 \pi}{\tau}\right)+4 \sum_{n=1}^{\infty} d(n) & K_{0}(2 \pi \tau n) \\
= & \tau^{-1}[\gamma-\log (4 \pi \tau)]+4 \tau^{-1} \sum_{n=1}^{\infty} d(n) K_{0}\left(\frac{2 \pi}{\tau} n\right) ;
\end{aligned}
$$

(3) Voronor's sum formula

$$
\begin{aligned}
-\frac{1}{4} f(0) & +\sum_{n=1}^{\infty} d(n) f(n)=\int_{0}^{\infty}(2 \gamma+\log x) f(x) d x \\
& +4 \sum_{n=1}^{\infty} d(n) \int_{0}^{\infty} f(x)\left[K_{0}\left(4 \pi(n x)^{1 / 2}\right)-\frac{\pi}{2} Y_{0}\left(4 \pi(n x)^{1 / 2}\right)\right] d x .
\end{aligned}
$$

In fact, these were obtained in [5] by specializing parameters in relations which were proved equivalent. In the present note, we give a proof of the statement $[5$, p. 63] that two other special cases of (3), namely

$$
\begin{aligned}
\frac{\rho}{2 \pi^{2}} \sum_{n=1}^{\infty} d(n) \frac{\log \left(\frac{n}{\rho}\right)}{n^{2}-\rho^{2}}= & \frac{\gamma}{4}+\frac{1}{8} \log \rho+\frac{1}{8 \pi^{2} \rho} \log \left(4 \pi^{2} \rho\right) \\
& +\sum_{n=1}^{\infty} d(n) K_{0}\left(4 \pi(n \rho)^{1 / 2}\right)
\end{aligned}
$$

and

Presented to the Society, September 3, 1965 under the title Some relations associated with Koshliakov's formula; received by the editors February 1, 1965. 


$$
\begin{aligned}
\rho \sum_{n=1}^{\infty} \frac{d(n)}{\rho^{2}+n^{2}}= & \frac{1}{4 \rho}+\gamma \pi+\frac{1}{2} \pi \log \rho \\
& +2 \pi \sum_{n=1}^{\infty} d(n)\left\{K_{0}\left(4 \pi(-i n \rho)^{1 / 2}\right)+K_{0}\left(4 \pi(i n \rho)^{1 / 2}\right)\right\}
\end{aligned}
$$

can also be considered equivalent to (3). Thus any one of the relations (1) through (5) implies the others. However, as in [5], we shall treat the problem in a somewhat generalized form.

In the course of our investigation, we shall need the following results:

$$
\begin{array}{cc}
\int_{0}^{\infty} x^{\rho-1} K_{0}(x y) d x=\frac{1}{4}\left(\frac{2}{y}\right)^{\rho}\left[\Gamma\left(\frac{\rho}{2}\right)\right]^{2}, & {[1, \text { p. 127], }} \\
\int_{0}^{\infty} K_{0}\left(\frac{a}{x}\right) K_{0}(x y) d x=\frac{\pi}{y} K_{0}\left(2(a y)^{1 / 2}\right), & {[1, \text { p. 146] }} \\
\int_{0}^{\infty} x K_{0}(a x) K_{0}(x y) d x=\frac{\log \left(\frac{a}{y}\right)}{a^{2}-y^{2}}, \\
\int_{0}^{\infty} \log x x^{\rho-1} K_{0}(x y) d x \\
=\frac{1}{4}\left(\frac{2}{y}\right)^{\rho}\left[\Gamma\left(\frac{\rho}{2}\right)\right]^{2}\left\{\log \left(\frac{2}{y}\right)+\psi\left(\frac{\rho}{2}\right)\right\} .
\end{array}
$$

(8) can easily be obtained from $[1$, p. 145] and (9) from (6) above.

(9a) $\int_{0}^{\infty} K_{0}(a x)\left[\frac{2}{\pi} K_{0}\left((x y)^{1 / 2}\right)-Y_{0}\left((x y)^{1 / 2}\right)\right] d x=\frac{1}{a} K_{0}\left(\frac{y}{4 a}\right)$

[5, p. 51].

1. We assume that

$$
\begin{array}{ll}
a_{1}, a_{2} \cdots ; & 0<\lambda_{1}<\lambda_{2} \cdots \rightarrow \infty, \\
b_{1}, b_{2} \cdots ; & 0<l_{1}<l_{2} \cdots \rightarrow \infty
\end{array}
$$

are four sequences of numbers such that the series

$$
\sum_{n=1}^{\infty} \frac{\left|a_{n}\right|}{\lambda_{n}^{2}} \text { and } \sum_{n=1}^{\infty} \frac{\left|b_{n}\right|}{l_{n}^{2}}
$$

are convergent.

(b) $\alpha, \alpha^{\prime}, \beta, \beta^{\prime}$ are four numbers such that the following relation holds 
(I)

$$
\begin{aligned}
\alpha[\gamma+\log (\pi \tau)] & -\alpha^{\prime}+\sum_{n=1}^{\infty} a_{n} K_{0}\left(2 \pi \lambda_{n} \tau\right) \\
= & \frac{1}{\tau}\left\{\beta\left[\gamma+\log \left(\frac{\pi}{\tau}\right)\right]-\beta^{\prime}+\sum_{n=1}^{\infty} b_{n} K_{0}\left(\frac{2 \pi l_{n}}{\tau}\right)\right\} .
\end{aligned}
$$

If we multiply both sides of (I) by $\tau K_{0}(2 \pi \tau y),(y>0)$, and integrate with regard to $\tau$ from zero to infinity, we get

(10)

$$
\begin{aligned}
{\left[\alpha \gamma+\alpha \log \pi-\alpha^{\prime}\right] } & \frac{1}{4 \pi^{2} y^{2}}+\alpha \frac{1}{4 \pi^{2} y^{2}}[-\log \pi y-\gamma] \\
& +\frac{1}{4 \pi^{2}} \sum_{n=1}^{\infty} a_{n} \frac{\log \left(\frac{\lambda_{n}}{y}\right)}{\lambda_{n}^{2}-y^{2}} \\
= & {\left[\beta \gamma+\beta \log \pi-\beta^{\prime}\right] \frac{1}{4 y}+\frac{\beta}{4 y}[\gamma+\log (4 \pi y)] } \\
& +\frac{1}{2 y} \sum_{n=1}^{\infty} b_{n} K_{0}\left(4 \pi\left(l_{n} y\right)^{1 / 2}\right) .
\end{aligned}
$$

The interchange of the order of summation and integration is justified because of absolute convergence.

We rewrite (10) as

$$
\begin{aligned}
& \frac{1}{2 \pi^{2} y}\left[-\alpha \log y-\alpha^{\prime}\right]+\frac{y}{2 \pi^{2}} \sum_{n=1}^{\infty} a_{n} \frac{\log \frac{\lambda_{n}}{y}}{\lambda_{n}^{2}-y^{2}} \\
& =\left[\beta \gamma+\frac{1}{2} \beta \log \left(4 \pi^{2} y\right)-\frac{1}{2} \beta^{\prime}\right]+\sum_{n=1}^{\infty} b_{n} K_{0}\left(4 \pi\left(l_{n} y\right)^{1 / 2}\right) .
\end{aligned}
$$

By analytic continuation (II) holds for all $y$ such that $-\pi<\arg y<\pi$. Likewise, if we multiply (I) by $\left(1 / \tau^{2}\right) K_{0}(2 \pi y / \tau)$ and integrate, we get

$$
\begin{gathered}
\left(\alpha \gamma+\frac{1}{2} \alpha \log \left(4 \pi^{2} y\right)-\frac{1}{2} \alpha^{\prime}\right)+\sum_{n=1}^{\infty} a_{n} K_{0}\left(4 \pi\left(\lambda_{n} y\right)^{1 / 2}\right) \\
=\frac{1}{2 \pi^{2} y}\left(-\beta \log y-\beta^{\prime}\right)+\frac{y}{2 \pi^{2}} \sum_{n=1}^{\infty} b_{n} \frac{\log \left(\frac{l_{n}}{y}\right)}{l_{n}^{2}-y^{2}} .
\end{gathered}
$$

If we replace $y$ by $e^{i x} y$ in (II), we get the relation 


$$
\begin{aligned}
\frac{1}{2 \pi^{2} y}\left[\alpha \log \left(y e^{i \pi}\right)+\alpha^{\prime}\right]+\frac{y}{2 \pi^{2}} \sum_{n=1}^{\infty} a_{n} \frac{\log \left(\frac{\lambda_{n}}{y e^{i \pi}}\right)}{y^{2}-\lambda_{n}^{2}} & \\
= & {\left[\beta \gamma+\frac{1}{2} \beta \log \left(4 \pi^{2} y e^{i \pi}\right)-\frac{1}{2} \beta^{\prime}\right]+\sum_{n=1}^{\infty} b_{n} K_{0}\left(4 \pi\left(l_{n} y e^{i \pi}\right)^{1 / 2}\right), } \\
& -2 \pi<\arg y<0 .
\end{aligned}
$$

Adding the corresponding sides of (II) and (11), we obtain

$$
\begin{aligned}
\frac{i \alpha}{2 \pi y}-\frac{i y}{2 \pi} \sum_{n=1}^{\infty} \frac{a_{n}}{y^{2}-\lambda_{n}^{2}} \\
(\mathrm{III})=2 \beta \gamma+\beta \log \left(4 \pi^{2} y\right)-\beta^{\prime}+\frac{1}{2} i \pi \beta \\
\quad+\sum_{n=1}^{\infty} b_{n}\left\{K_{0}\left(4 \pi\left(l_{n} y\right)^{1 / 2}\right)+K_{0}\left(4 \pi\left(l_{n} y e^{i \pi}\right)^{1 / 2}\right)\right\},-\pi<\arg y<0 .
\end{aligned}
$$

The corresponding relation derived from $\left(\mathrm{II}^{\prime}\right)$ is

$$
\begin{aligned}
2 \alpha \gamma+\alpha \log \left(4 \pi^{2} y\right) & -\alpha^{\prime}+\frac{1}{2} i \pi \alpha \\
& +\sum_{n=1}^{\infty} a_{n}\left\{K_{0}\left(4 \pi\left(\lambda_{n} y\right)^{1 / 2}\right)+K_{0}\left(4 \pi\left(\lambda_{n} y e^{i \pi}\right)^{1 / 2}\right)\right\} \\
= & \frac{i \beta}{2 \pi y}-\frac{i y}{2 \pi} \sum_{n=1}^{\infty} \frac{b_{n}}{y^{2}-l_{n}^{2}}, \quad-\pi<\arg y<0 .
\end{aligned}
$$

2. Let

$$
\sigma(y)=\frac{1}{2 \pi i}\left\{\sum_{n=1}^{\infty} a_{n} \frac{2 y}{y^{2}-\lambda_{n}^{2}}-2 \frac{\alpha}{y}\right\} .
$$

Then from (III)

$$
\begin{aligned}
\frac{1}{2} \sigma(y)= & 2 \beta \gamma+\beta \log \left(4 \pi^{2} y\right)-\beta^{\prime}+\frac{1}{2} i \pi \beta \\
& +\sum_{n=1}^{\infty} b_{n}\left\{K_{0}\left(4 \pi\left(l_{n} y\right)^{1 / 2}\right)+K_{0}\left(4 \pi\left(l_{n} y e^{i \pi}\right)^{1 / 2}\right)\right\}, \quad-\pi<\arg y<0, \\
= & -\left[2 \beta \gamma+\beta \log \left(4 \pi^{2} y\right)-\beta^{\prime}\right]+\frac{1}{2} i \pi \beta \\
& -\sum_{n=1}^{\infty} b_{n}\left\{K_{0}\left(4 \pi\left(l_{n} y\right)^{1 / 2}\right)+K_{0}\left(4 \pi\left(l_{n} y e^{-i \pi}\right)^{1 / 2}\right)\right\}, \quad 0<\arg y<\pi .
\end{aligned}
$$

Let $\phi(y)$ be a function of the complex variable $y=u+i v$, regular in a strip $u \geqq 0,|v| \leqq \delta$, for some $\delta>0$ and satisfying the following conditions: 
(i) $\int_{0}^{\infty}|\phi(u+i v)| d u$ and $\int_{0}^{\infty} \log (u+i v) \phi(u+i v) d u$ converge in

$$
-\delta<v<\delta .
$$

(ii) There exists a sequence of the numbers $u_{n}$ such that

(13) $\lim _{n \rightarrow \infty} \sigma\left(u_{n}+i v\right) \phi\left(u_{n}+i v\right)=0$ uniformly in $-\delta<v<\delta$.

Since $\sigma(y)$ is analytic except for simple poles at zero and $\pm \lambda_{n}$, $n=1,2,3, \cdots$, we obtain by Cauchy's theorem and (13)

$$
\sum_{n=1}^{\infty} a_{n} \phi\left(\lambda_{n}\right)=\int_{C} \sigma(y) \phi(y) d y
$$

where $C$ is the contour shown. Now we let $\epsilon \rightarrow 0$.

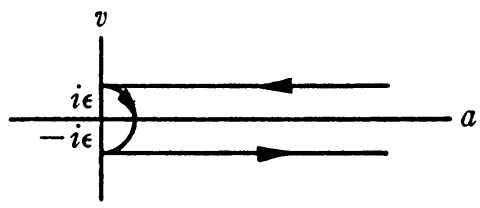

$$
0<\epsilon<\min \left(\delta, \lambda_{1}\right) \text {. }
$$

Thus

$$
\begin{aligned}
& \sum_{n=1}^{\infty} a_{n} \phi\left(\lambda_{n}\right) \\
& =\alpha \phi(0)+4 \int_{0}^{\infty}\left[2 \beta \gamma-\beta^{\prime}+\beta \log \left(4 \pi^{2} u\right)\right] \phi(u) d u \\
& +\lim _{\epsilon \rightarrow 0}\left\{2 \int_{-i \epsilon}^{\infty-i \epsilon} \phi(y) \sum_{n=1}^{\infty} b_{n}\left[K_{0}\left(4 \pi\left(l_{n} y\right)^{1 / 2}\right)+K_{0}\left(4 \pi\left(l_{n} y e^{i \pi}\right)^{1 / 2}\right)\right] d y\right. \\
& \left.+2 \int_{i \epsilon}^{\infty+i \epsilon} \phi(y) \sum_{n=1}^{\infty} b_{n}\left[K_{0}\left(4 \pi\left(l_{n} y\right)^{1 / 2}\right)+K_{0}\left(4 \pi\left(l_{n} y e^{-i \pi}\right)^{1 / 2}\right)\right] d y\right\} .
\end{aligned}
$$

Let $\phi(y)$ be such that we can interchange the order of summation and integration. Then proceeding to the limit as $\epsilon \rightarrow 0$; and using

$$
K_{0}\left(y e^{i \pi / 2}\right)+K_{0}\left(y e^{-i \pi / 2}\right)=-\pi Y_{0}(y),
$$

we obtain 


$$
\begin{aligned}
-\alpha \phi(0) & +\sum_{n=1}^{\infty} a_{n} \phi\left(\lambda_{n}\right)=4 \int_{0}^{\infty}\left[2 \beta \gamma-\beta^{\prime}+\beta \log \left(4 \pi^{2} u\right)\right] \phi(u) d u \\
& +4 \sum_{n=1}^{\infty} b_{n} \int_{0}^{\infty}\left[K_{0}\left(4 \pi\left(l_{n} u\right)^{1 / 2}\right)-\frac{\pi}{2} Y_{0}\left(4 \pi\left(l_{n} u\right)^{1 / 2}\right)\right] \phi(u) d u .
\end{aligned}
$$

Likewise from $\left(\mathrm{III}^{\prime}\right)$, we get the Sum-formula

$$
-\beta \phi(0)+\sum_{n=1}^{\infty} b_{n} \phi\left(l_{n}\right)=4 \int_{0}^{\infty}\left[2 \alpha \gamma-\alpha^{\prime}+\alpha \log \left(4 \pi^{2} u\right)\right]_{\phi}(u) d u
$$

$$
+4 \sum_{n=1}^{\infty} a_{n} \int_{0}^{\infty}\left[K_{0}\left(4 \pi\left(\lambda_{n} u\right)^{1 / 2}\right)-\frac{\pi}{2} Y_{0}\left(4 \pi\left(\lambda_{n} u\right)^{1 / 2}\right)\right] \phi(u) d u .
$$

If in (IV) or $\left(I V^{\prime}\right)$, we let

$$
\begin{aligned}
& \phi(u)=K_{0}(2 \pi u \tau)-K_{0}(2 \pi u), \quad 0<u<\infty, \\
& \phi(0)=\log \frac{1}{\tau},
\end{aligned}
$$

and further assume that

(c)

$$
\begin{aligned}
\alpha[\gamma+\log \pi]-\alpha^{\prime}+\sum_{n=1}^{\infty} a_{n} K_{0}\left(2 \pi \lambda_{n}\right) & \\
= & \beta[\gamma+\log \pi]-\beta^{\prime}+\sum_{n=1}^{\infty} b_{n} K_{0}\left(2 \pi l_{n}\right) .
\end{aligned}
$$

Then using (6), (9), (9a), we get the relation (I).

Thus we have proved the following:

1. Under condition (a), each one of the six relations (II), (II'), (III), (III'), (IV), (IV') is a consequence of (I).

2. Under conditions (a) and (c), the relation (I) through (IV') are equivalent to each other.

If we set

$$
\begin{array}{rlrl}
a_{n} & =b_{n}=d(n) ; & \lambda_{n}=l_{n}=n, \\
\alpha=\beta=\frac{1}{4} ; & \alpha^{\prime}=\beta^{\prime}=\frac{1}{2} \log (2 \pi),
\end{array}
$$

in relations (I)-(IV') and note that conditions (a) and (c) are satisfied, we get the corresponding special cases mentioned in the introduction.

3. Now we study the functions $f(s)$ and $g(s)$ defined by: 


$$
\begin{array}{ll}
f(s)=\sum_{n=1}^{\infty} \frac{a_{n}}{\lambda_{n}^{s}}, & \operatorname{Re} s \geqq 2, \\
g(s)=\sum_{n=1}^{\infty} \frac{b_{n}}{l_{n}^{2}}, & \operatorname{Re} s \geqq 2,
\end{array}
$$

when conditions (a) and (b) hold.

Let

$\mu(y)=\sum_{n=1}^{\infty} b_{n}\left\{K_{0}\left(4 \pi\left(l_{n} y\right)^{1 / 2}\right)+K_{0}\left(4 \pi\left(l_{n} y e^{i \pi}\right)^{1 / 2}\right)\right\}, \quad-\pi<\arg y<0$,

then

$\mu\left(e^{-i \pi} y\right)=\sum_{n=1}^{\infty} b_{n}\left\{K_{0}\left(4 \pi\left(l_{n} y e^{-i \pi}\right)^{1 / 2}\right)+K_{0}\left(4 \pi\left(l_{n} y\right)^{1 / 2}\right)\right\}, \quad 0<\arg y<\pi$.

We denote the contour opposite as $C_{\nu}$.

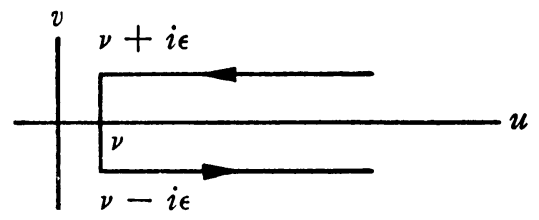

For $0<\nu<\lambda_{1}$ and $\phi(y)=y^{-8}$, Re $s \geqq 2$, we obtain

$$
\begin{aligned}
f(s)= & \sum_{n=1}^{\infty} \frac{a_{n}}{\lambda_{n}^{s}}=\int_{C_{\nu}} \sigma(y) y^{-s} d y \\
= & 4 \int_{\nu}^{\infty}\left[2 \beta \gamma-\beta^{\prime}+\beta \log \left(4 \pi^{2} u\right)\right] u^{-s} d u+2 \int_{\nu}^{\nu-i \epsilon} \frac{\mu(y)}{y^{s}} d y \\
& +2 \int_{\nu}^{\nu+i \epsilon} \frac{\mu\left(e^{-i \pi} y\right)}{y^{s}} d y+2 \int_{\nu-i \epsilon}^{\infty-i \epsilon} \frac{\mu(y)}{y^{s}} d y+2 \int_{\nu+i \epsilon}^{\infty+i \epsilon} \frac{\mu\left(e^{-i \pi} y\right)}{y^{s}} d y .
\end{aligned}
$$

Making use of the fact that

$$
\sum_{n=1}^{\infty} b_{n} K_{0}\left(4 \pi\left(l_{n} z\right)^{1 / 2}\right)=O\left(\exp \left[-4 \pi\left(l_{1} \rho\right)^{1 / 2} \cos \theta / 2\right)\right.
$$

where $z=\rho e^{i \theta}$, we replace the path of integration in the last two integrals by ones at right-angles to them. Thus 


$$
\begin{aligned}
f(s)= & 4\left[2 \beta \gamma-\beta^{\prime}+\beta \log \left(4 \pi^{2}\right)\right] \frac{\nu^{1-s}}{s-1}+4 \beta \log \nu \frac{\nu^{1-s}}{s-1} \\
& +4 \beta \frac{\nu^{1-s}}{(s-1)^{2}}+2 \int_{\nu}^{\nu-i \infty} \frac{\mu(y)}{y^{\imath}} d y+2 \int_{\nu}^{\nu+i \infty} \frac{\mu\left(e^{-i \pi} y\right)}{y} d y .
\end{aligned}
$$

But the above integrals define a function of $s$ which is regular for all finite values of $s$. Therefore $f(s)$ is analytic and single valued in the whole $s$-plane except perhaps at $s=1$, where it may have a pole of the second order, with the principal part

$$
\frac{8 \beta \gamma-4 \beta^{\prime}+4 \beta \log \left(4 \pi^{2}\right)}{s-1}+\frac{4 \beta}{(s-1)^{2}} .
$$

Likewise, using (III'), which is a consequence of (b), we can show that $g(s)$ has the same properties as $f(s)$, and that the principal part of $g(s)$ at $s=1$ is

$$
\frac{4 \alpha}{(s-1)^{2}}+\frac{8 \alpha \gamma-4 \alpha^{\prime}+4 \alpha \log \left(4 \pi^{2}\right)}{s-1} .
$$

Now if we proceed to the limit as $\nu \rightarrow 0$ in $\operatorname{Re} s<1$, we obtain from (14)

$$
\begin{aligned}
f(s) & =2 \int_{0}^{-i \infty} \frac{\mu(y)}{y^{s}} d y+2 \int_{0}^{i \infty} \frac{\mu\left(e^{-i \pi} y\right)}{y^{s}} d y \\
& =4 \sin \left(\frac{\pi}{2} s\right) \int_{0}^{\infty} \frac{\mu\left(e^{-i \pi / 2} t\right)}{t^{s}} d t .
\end{aligned}
$$

This gives the integral representation of $f(s)$ in the half $s$-plane.

If we interchange the order of integration and summation in (15) and use

$$
\int_{0}^{\infty} K_{0}\left(a t^{1 / 2}\right) t^{-s} d t=a^{2 s-2} 2^{1-2 s}[\Gamma(1-s)]^{2}, \quad \operatorname{Re} s<1 ;|\arg a|<\frac{\pi}{2},
$$

we obtain

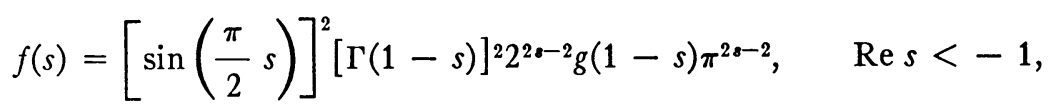

which can be written as

$$
\pi^{-s}\left[\Gamma\left(\frac{s}{2}\right)\right]^{2} f(s)=\pi^{-1+s}\left[\Gamma\left(\frac{1-s}{2}\right)\right]^{2} g(1-s)
$$


and by analytic continuation equality (16) holds for all $s$.

Finally, expanding both sides of (16) around $s=0$ and $s=1$ respectively, we find that

$$
\alpha=f(0), \quad \alpha^{\prime}=f^{\prime}(0), \quad \beta=g(0), \quad \beta^{\prime}=g^{\prime}(0) .
$$

Thus we have proved the following: Under conditions (a) and (b), $(s-1)^{2} f(s)$ and $(s-1)^{2} g(s)$ are entire functions; $f(s)$ and $g(s)$ satisfy the functional equation (16) and further $\alpha, \alpha^{\prime}, \beta, \beta^{\prime}$ are related to these functions as given in (17).

Acknowledgment. Professor F. Oberhettinger suggested this problem and gave every encouragement. To him I wish to express my gratitude.

\section{REFERENCES}

1. A. Erdélyi, Tables of integral transforms, Vol. 2, McGraw-Hill, New York, 1954.

2. H. Hamburger, Ueber einige Beziehungen die mit der Funktional gleichung der Zeta Funktion aequivalent Sind, Math. Ann. 85 (1922), 129-140.

3. N. S. Koshliakov, Application of the theory of sum formulae to the investigation of a class of one valued analytical functions in the theory of numbers, Messenger of Math. 58 (1928), 1-23.

4. - On Voronot's sum formula, Messenger of Math. 58 (1928), 30-32.

5. K. Soni, On some relations which are equivalent to the functional equations of the type of Riemann's zeta function and tables of divisor transforms, $\mathrm{Ph}$. D. Thesis, Oregon State University, Corvallis, Oregon, 1964.

ENDICOTT, NEW YORK 